\title{
Bereavement
}

Grief after the death of a child is described as the most painful and enduring. It is also associated with a higher risk of pathological grief. Parents suffer multiple losses. Siblings suffer too and may have difficulty adjusting; they often feel isolated and neglected, as their parents can spare little energy or emotion for them.

Helping the bereaved family involves

- Support and assessment through the tasks of normal mourning-Most families do not need specialist counselling but benefit from general support and reassurance, supplied if possible by those who have known the family through the illness

- Information-Such as support groups and the Child Death Helpline (freephone 0800 282986); many parents value the opportunity of talking with others who have also experienced the death of a child

- Referral for specialist bereavement counselling if needed

- Gradual withdrawal of contact.

Ann Goldman is CLIC consultant in palliative care at Great Ormond Street Hospital for Children, London.

The ABC of palliative care is edited by Marie Fallon, Marie Curie senior lecturer in palliative medicine, Beatson Oncology Centre, Western Infirmary, Glasgow, and Bill O'Neill , science and research adviser, British Medical Association, BMA House, London. It will be published as a book in June 1998.

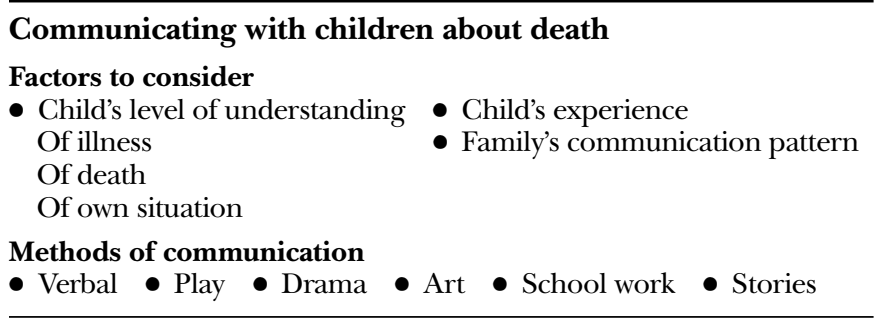

\section{The loss of a child}

- Multiple losses for parents The child who has died Their dreams and hopes Their own immortality Their role as parents

\section{Further reading}

- ACT, Royal College of Paediatrics and Child Health guide to the development of children's palliative care services. 1997. Available from ACT, 65 St Michael's Hill, Bristol BS2 8DZ (Tel 0117922 1556, Fax 01179304707$.

- Royal College of Paediatrics and Child Health. Prevention and control of pain in children: a manual for health care professionals. London, BMJ Publishing, 1997. (Tel 0171383 6185, Fax 0171383 6662.)

\section{Lesson of the week Early diagnosis of pyoderma gangrenosum is important
to prevent disfigurement}

\author{
A J Harris, P Regan, S Burge
}

\section{Pyoderma gangrenosum should be suspected in patients with non-healing ulcers}

Department of Dermatology, Stoke Mandeville NHS Trust,

Aylesbury,

Buckinghamshire

HP21 8AL

A J Harris,

dermatology registrar

S Burge,

consultant

dermatologist

continued over

BMJ 1998;316:52-3
Pyoderma gangrenosum is a destructive inflammatory disease that responds well to immunosuppression, though surgery may exacerbate the lesions. Diagnosis is based on clinical findings. We report the case of a 24 year old man with non-healing ulcers to highlight the importance of considering pyoderma gangrenosum in patients with this symptom.

\section{Case report}

A previously fit 24 year old white man was referred to the department of dermatology with a 12 month history of a painful, enlarging ulcer on the right side of his jaw. A year earlier his general practitioner had excised a cyst from his right ear lobe, but the wound had dehisced and become ulcerated. Four attempts were made to close the wound surgically over the next year. On the first and second attempts the wound was debrided and closed immediately. Because the defect was too large to close on the third and fourth attempts a skin graft was used. On each occasion the wound failed to heal and the ulcer grew. Skin donor sites on the thighs healed normally. $x$ Ray films of the mandible were normal. A decaying tooth was extracted from the patient's left lower jaw and the tooth socket also failed to heal.

Cultures of debrided tissue were negative for bacteria, mycobacteria, and fungi. Cultures from the tooth socket were also sterile. Histological examination identified a non-specific mixed inflammatory cell infiltrate with areas of necrosis. The surgeons suspected dermatitis artefacta, and referred the patient to a dermatologist.

When the patient was examined by the dermatologist the ulcer extended over the angle of the jaw and affected the right ear (fig 1). It had a haemorrhagic base with indurated, violaceous, undermined borders. Pus exuded from the ulcerated cartilage of the right ear. The ear lobe was partially destroyed. A small ulcer on the nape of the neck, an ulcerated left nipple, and an ulcer where the tooth had been extracted were also found. All had been precipitated by trauma.

A diagnosis of pyoderma gangrenosum was considered. The patient had a normal full blood count and erythrocyte sedimentation rate. Urea, electrolyte, and $\mathrm{C}$ reactive protein concentrations were normal, and liver function tests gave normal results. Tests for rheumatoid factor, antinuclear antibodies, and 
antineutrophil cytoplasmic antibodies gave negative results. No associated disease was identified.

The patient was treated with $40 \mathrm{mg}$ prednisolone and $200 \mathrm{mg}$ minocycline daily and three bolus doses of intravenous methylprednisolone $1 \mathrm{~g}$ given over 3 weeks. Clobetasol propionate, a topical steroid, was applied to the ulcer for 6 weeks. The ulcer on the patient's jaw decreased in size and the edges flattened within 6 weeks. After six weeks the other ulcerated areas (neck, left nipple, and tooth socket) healed completely, but the patient became increasingly cushingoid. A daily dose of cyclosporin $3 \mathrm{mg}$ per $\mathrm{kg}$ of body weight was added to the regimen for the duration of the treatment. The ulcer healed after 5 months of treatment, but a disfiguring scar remained (fig 2). Immunosuppression was withdrawn slowly.

\section{Discussion}

The diagnosis of pyoderma gangrenosum is based on clinical findings, and our patient had many of the classic features. Pyoderma gangrenosum generally presents as a rapidly enlarging, painful ulcer with purple, undermined edges and a necrotic, haemorrhagic base. Pustular, follicular, nodular, bullous, or cribriform lesions have also been described. ${ }^{1}$ Many patients have more than one lesion. The importance of examining the patient's skin is shown in this case in which minor trauma had induced persistent ulcers at other sites. In $15 \%$ to $40 \%$ of cases of pyoderma gangrenosum patients develop ulcers at sites of quite minor trauma ${ }^{2}$-so surgery is contraindicated.

The leg is most commonly affected by pyoderma gangrenosum, and the atypical site described in our case may have delayed diagnosis. The head and neck are rarely affected, but when they are pyoderma gangrenosum may be reported as malignant pyoderma; the two diseases are now thought to be synonymous. ${ }^{145}$

Other causes of ulceration should be excluded. The ulcers seen in dermatitis artefacta have well defined, angular shapes without undermined edges. Histological findings in pyoderma gangrenosum are nonspecific, ${ }^{6}$ but biopsy can exclude other disease, such as malignancy. Tissues should be cultured to exclude fungal or mycobacterial infection in granulomas. Infection must be excluded before immunosuppressive treatment is started.

The uncomplicated medical history of our patient is not unusual, only $50 \%$ of patients with pyoderma gangrenosum have related medical conditions such as inflammatory bowel disease, rheumatoid arthritis, and haematological malignancies. ${ }^{27}$ The diagnosis of pyoderma gangrenosum should be considered sooner in patients with related medical problems. The association between pyoderma gangrenosum on the face and Wegener's granulomatosis is well known; Wegener's granulomatosis should be specifically excluded in all cases of pyoderma gangrenosum affecting the head and neck. ${ }^{8}$

Repeated unsuccessful attempts at surgical closure or grafting of wounds is not uncommon in cases in which the diagnosis of pyoderma gangrenosum is delayed. Retrospective diagnosis of such cases is often easy, but an early diagnosis is essential to allow immu-

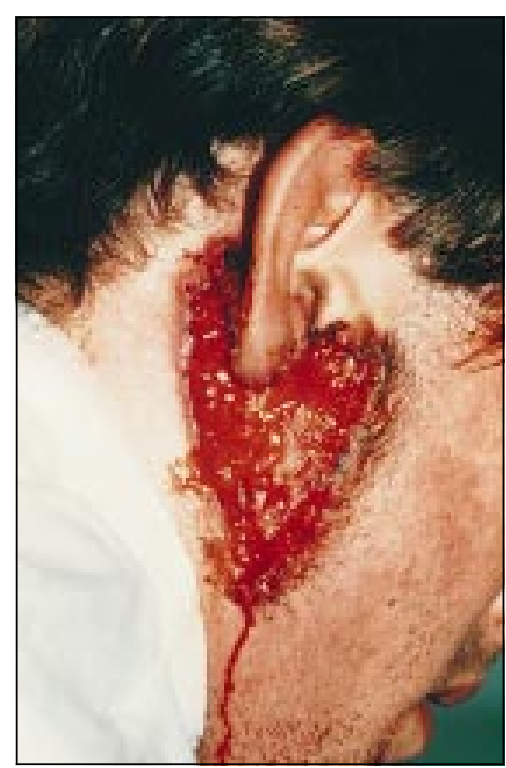

Fig 1 Ulcer at time patient referred to dermatologist

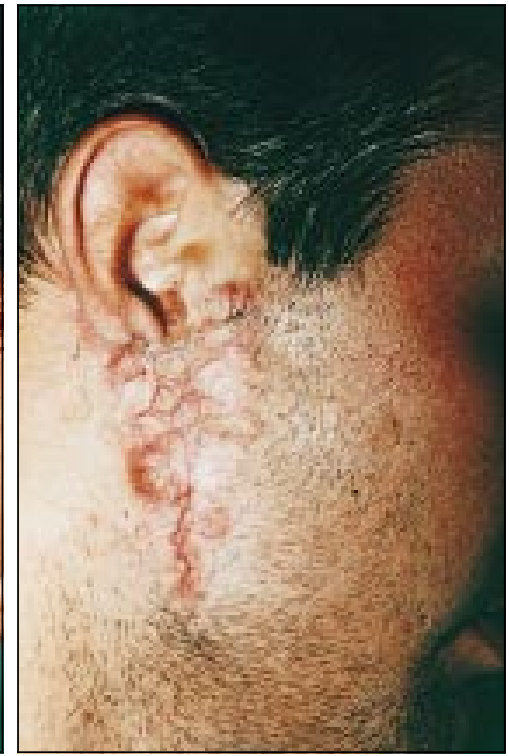

Fig 2 Patient after 5 months of treatment nosuppression treatment to start once infection has been excluded. Prompt treatment minimises scarring. First line treatment consists of oral steroids sometimes combined with steroid sparing agents such as dapsone or minocycline. In less severe cases steroids may be given topically or intralesionally instead of orally. ${ }^{9}$ When the diagnosis is unclear a rapid response to oral steroids may be diagnostic. Cyclosporin is useful in stubborn cases. ${ }^{9}$

Pyoderma gangrenosum is an important differential diagnosis in patients with growing painful ulcers on any part of the body. Potentially harmful surgery should be avoided. Early treatment with immunosuppression is essential to control the disease and prevent permanent scarring.

1 Snyder RA. Pyoderma gangrenosum involving the head and neck. Arch Dermatol 1986;122:295-302.

2 Fowler JF, Callen JP. Pyoderma gangrenosum. Dermatol Clin 1983:1: 615-22.

3 Prystowsky JH, Kahn SN, Lazarus GS. Present status of pyoderma gangrenosum: review of 21 cases. Arch Dermatol 1989;125:57-64.

4 Malkinson FD. Pyoderma gangrenosum vs malignant pyoderma. Arch Dermatol 1987;123:333-7.

5 Wernikoff S, Merritt C, Briggaman R, Woodley DT. Malignant pyoderma or pyoderma gangrenosum of the head and neck. Arch Dermatol 1987;123:371-5.

6 Su WPD, Schroeter HO, Perry HO, Powell FC. Histopathologic and immunopathologic study of pyoderma gangrenosum. Cutan Pathol 1986;13:323-30.

7 Hickman JG, Lazarus GS. Pyoderma gangrenosum: a reappraisal of associated systemic diseases. Br J Dermatol 1980;102:235-7.

8 Micall G, Cook B. Cephalic pyoderma gangrenosum (PG)-like lesions as a presenting sign of Wegener's granulomatosis. Int J Dermatol a presenting $1994 ; 33: 477-80$.

9 Chow RKP, Ho VC. Treatment of pyoderma gangrenosum. J Am Acad Dermatol 1996; 34:1047-60.

(Accepted 3 September 1997)

\section{Endpiece}

\section{Asher on treatment}

A remedy which is known to work, though nobody knows why, is preferable to a remedy which has the support of theory without the confirmation of practice.

From A Sense of Asher, selected by Ruth Holland (BMA Publications, 1984)
Department of Plastic Surgery, Stoke Mandeville NHS Trust

P Regan, consultant plastic surgeon

Correspondence to: Dr A J Harris, Department of Dermatology, Guy's Hospital, London SE1 9RT 